\title{
Multiple interacting planar cracks in an anisotropic multilayered medium under an antiplane shear stress: a hypersingular integral approach
}

\author{
W. T. Ang ${ }^{\star a}$ \& A. B. Gumel ${ }^{b}$ \\ ${ }^{a}$ Faculty of Engineering, ${ }^{b}$ Faculty of Information Technology, Universiti Malaysia Sarawak, 94300 Kota Samarahan, Malaysia
}

(Received 21 June 1996; revised version received 13 December 1996; accepted 10 January 1997)

\begin{abstract}
The problem of determining the stress field around an arbitrary number of arbitrarily-located planar cracks in an anisotropic elastic half-space which adheres perfectly to an infinitely-long elastic strip is considered. The strip is made up of several layers of anisotropic materials which are perfectly bonded to one another. The multilayered medium is assumed to undergo an antiplane deformation. Suitable integral expressions are used to represent the displacement and the stress, leading to a system of hypersingular integral equations to be solved. For a specific example of the problem, which involves particular transversely-isotropic materials, the hypersingular integral equations are solved numerically, in order to calculate the relevant crack tip stress intensity factors. (C) Elsevier Science Ltd
\end{abstract}

Keywords: cracks, multilayered half-space, hypersingular integral formulation.

\section{INTRODUCTION}

Composite and anisotropic structures can now be found in an increasingly wider range of applications in modern technology. For example, media which are made up of many very fine layers are used in optical recording, artificial implants are surgically inserted into human bones which undergo orthopaedic treatments, and synthetic materials (e.g. delta wood, plywood and fabric laminate) which exhibit anisotropic behaviours are employed in the construction of modern aircraft. There is therefore the need to assess the reliability and integrity of these structures. Consequently, many researchers have given serious attention to the task of analysing the stress in anisotropic layered materials, particularly those containing cracks (e.g. Lahiri et al. ${ }^{1}$ Ang and Clements, ${ }^{2}$ Ang, ${ }^{3,4}$ Clements, ${ }^{5}$ Clements et al. $^{6}$ and Willis ${ }^{7}$ ).

In the present paper, we calculate the stress field around multiple planar cracks in an anisotropic elastic half-space which adheres perfectly to an infinitelylong elastic strip. The strip is made up of several layers of anisotropic materials which are perfectly

*To whom correspondence should be addressed. bonded to one another. The cracks are assumed to become traction-free under the action of an internal antiplane shear stress.

The problem under consideration may be of interest to practical situations in which cracked structures are patched or reinforced with different materials, such as in the repairs of aircraft. It is also useful for studying the interaction of cracks in laminates which are made up of a large number of layers.

By choosing a suitable integral representation for the displacement, the task of calculating the stress distribution around the cracks is eventually reduced to solving a system of hypersingular integral equations. The unknown functions in the integral equations are the 'crack-opening displacements'. The integral equations can be solved numerically using a collocation technique. For a specific example of interest, which involves particular transversely-isotropic materials, we solve the integral equations numerically to compute the relevant crack tip stress intensity factors.

\section{THE PROBLEM}

With reference to an $0 x_{1} x_{2} x_{3}$ Cartesian coordinate 